\title{
A bulk-micromachined corner cube retroreflector with piezoelectric micro-cantilevers
}

\author{
Jongcheol Park and Jae Yeong Park
}

\begin{abstract}
A piezoelectrically actuated corner cube retroreflector (CCR) has been investigated for free space optical communications. The proposed CCR consisted of two mutually orthogonal bulk-micromachined mirror assembled with piezoelectrically actuated horizontal mirror. The vertical mirrors were fabricated by using anisotropic wet-etching of double silicon-on-insulator (SOI) wafer and horizontal mirror was supported by two stress-compensating and one actuating lead zirconate titanate (PZT) micro-cantilevers. The fabricated CCRs exhibited angular displacement of $1.87^{\circ}$ at 5 volts and switching times of $276 \mu \mathrm{s}$. It also exhibited a good cut-off frequency of $2.5 \mathrm{kHz}$ which can be digitally modulated up to about $5 \mathrm{~kb} / \mathrm{s}$.
\end{abstract}

Keywords: Corner cube retroreflectors (CCR); Optical mirrors; Piezoelectric actuators; Micro-cantilevers; Anisotropic silicon etching

\section{Introduction}

A corner cube retroreflector (CCR) has been developed as an optical passive transmitter in wireless optical communication with low power consumption [1]. While the CCR does not have a light source, it can transmit the data to the source by digitally modulated reflection of the incident light. It is comprised of two mutually orthogonal vertical mirrors and horizontal mirror with the magnetic or electro-static actuator. The actuator is utilized to form the angular displacement of the horizontal mirror. The electrostatic actuators have been commonly used due to their simple working principles and structures [2-5]. However, the electro-static actuator still needs high driving voltage to obtain the large angular displacement. Two mutually orthogonal vertical mirrors for the CCRs have successfully been fabricated using surface micromachining technique [2-5]. However, the alignment of two mirrors has been limited due to the curvature of the fabricated mirrors from the asymmetric film stresses and a manual assembling of two mirrors. In order to

*Correspondence: jaepark@kw.ac.kr

Department of Electronic Engineering, Kwangwoon University,

447-1 Wolgye-dong, Nowon-gu, Seoul 139-701, Republic of Korea improve the flatness and alignment of the mirrors, bonded silicon-on-insulator (BSOI) with structurally-assisted and assembled or self-assembled structure was utilized $[3,4]$. While they have presented good feasibility, it is not easy to obtain the accurate angular alignment to form mutually orthogonal mirror surfaces. In this study, a silicon bulk micromachined CCR was investigated with ultra-low voltage operation and negligible power consumption [6]. It was comprised of the bulk-micromachined silicon vertical mirror and silicon nitride horizontal mirror with piezoelectric cantilever actuator. For achieving good surface roughness, accurate angular alignment, and mass productivity of the vertical mirror, a new fabrication process was developed using a double-SOI wafer and anisotropic $\mathrm{KOH}$ etching technique. For obtaining a large displacement at low induced voltage and minimizing the initial angular displacement of the horizontal mirror, the piezoelectric micro-cantilever actuator and supports were new1y applied.

\section{Findings}

The PZT micro-cantilever was utilized as an actuator for the horizontal mirror to obtain large angular dis-

\section{是 Springer}

(C) 2013 Park and Park; licensee Springer. This is an Open Access article distributed under the terms of the Creative Commons Attribution License (http://creativecommons.org/licenses/by/2.0), which permits unrestricted use, distribution, and reproduction in any medium, provided the original work is properly cited. 
placement. However, the PZT cantilever has an initial bending due to its asymmetric film stress in multilayer structure. The initial bending of PZT cantilever introduces the angular misalignment between the horizontal and vertical mirrors. Since there is difficulty in the control of the residual stress in the deposition of PZT thin film, it might be serious problem for the MEMS CCR because the colinear differential scattering cross section (CDSCS) of MEMS CCR is affected by the radius of curvature and angular misalignment of mirrors [5]. Especially, the maximum angular misalignment of mirror should be below $0.055^{\circ}$ to communicated over free space and $0.11^{\circ}$ or more angular misalignment is sufficient to switch off the CCR. Therefore, the stress compensated structure of the PZT cantilevers was investigated for the horizontal mirror. Figure 1 presents the schematic drawing of the proposed horizontal mirror with the PZT microcantilevers for MEMS CCR. As shown in Figure 1 (a), the horizontal mirror was equally suspended by two supporting and one actuating PZT cantilevers with torsional meander springs. These two supporting PZT cantilevers were utilized to improve the angular alignment of the horizontal mirror by compensating the initial bending of the actuating PZT cantilever after the fabrication. Thus, the proposed CCR has three mutually orthogonal mirrors to reflect the incident light to the source as shown in Figure 1 (a). When the angular misalignment is occured by actuating cantilever as shown in Figure 1 (b), the incident light is scattered away from the source. Therefore, the proposed MEMS CCR can transmit the on-offkeyed digital siginal to the source as a passive optical transmitter.

The vertical mirror should have two mutually orthogonal reflective surfaces with accurate angular alignment and good surface roughness. Figure $2(\mathrm{a}-\mathrm{d})$ shows the fabrication procedure to obtain the two mutually orthogonal reflective surfaces with accurate angular alignment. A double-SOI wafer with a silicon spacer was used to fabricate the cross shaped vertical silicon mirror. The double-SOI wafer was comprised of two silicon wafers with thickness of $300 \mu \mathrm{m}$, buried oxide layers of $1 \mu \mathrm{m}$ in thickness, and a silicon spacer with thickness of $30 \mu \mathrm{m}$. On the double-SOI wafer, silicon nitride was deposited as a masking layer for $\mathrm{KOH}$ wet etching. Firstly, top and bottom SiNx layers were sequentially patterned using the same mask with parallel lines to $<111>$ direction. In order to obtain the symmetrically formed vertical comb structure with high aspect ratio, the double-SOI wafer was etched down to $300 \mu \mathrm{m}$ as far as by using $\mathrm{KOH}$ solution and the buried oxide was used as an etch stop layer. The $\mathrm{KOH}$ etchant was optimized to have $40{ }_{w t} \%$ of concentration and $70^{\circ} \mathrm{C}$ of processing temperature to minimize surface roughness of the fabricated silicon mirror structure. To obtain the cross shaped vertical mirror, the etched double-SOI wafer was carefully sawed in the perpendicular direction of the formed structures and finally rotated by $90^{\circ}$. In order to improve the reflectivity of the vertical mirror, $800 \AA$ of gold thin film was sputtered on the vertical silicon mirror surfaces.

Figure $2(\mathrm{e}-\mathrm{f})$ shows the fabrication process for the horizontally actuated mirror. Firstly, low stress SiNx layer of $1 \mu \mathrm{m}$ in thickness was deposited on a silicon substrate and then Ti/Pt bottom electrode, PZT, and Pt top electrode were sequentially deposited to have a thickness of $20 \mathrm{~nm} / 120 \mathrm{~nm}, 500 \mathrm{~nm}$, and $100 \mathrm{~nm}$, respectively. The PZT film was formed using spin-casting and annealing processes. The supporting and actuating PZT cantilevers were defined through the dry etching of $\mathrm{Pt} / \mathrm{PZT} / \mathrm{Pt}$ thin film. The horizontal mirrors with a size of $150 \times 150 \mu \mathrm{m}^{2}$

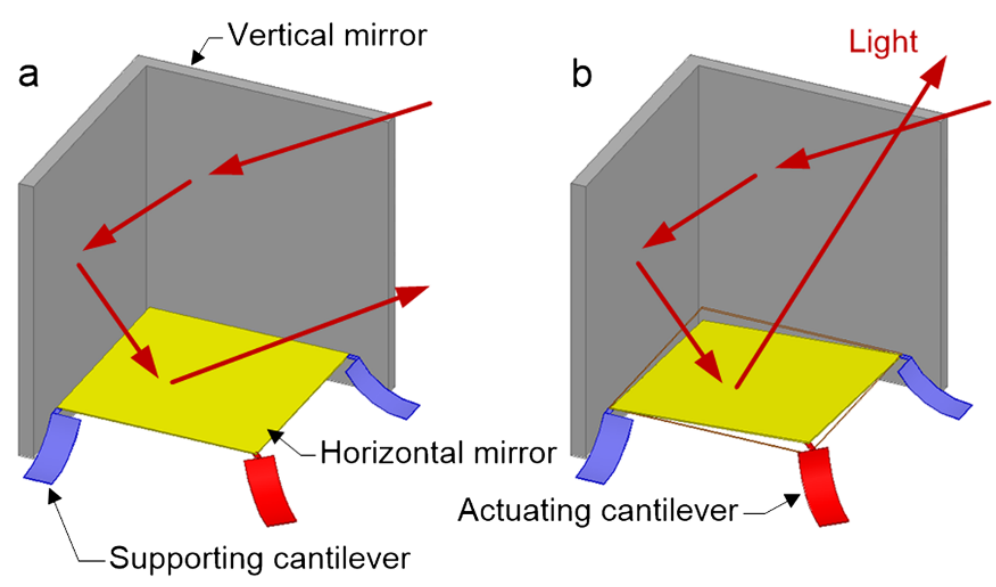

Figure 1 Schematic drawing of the proposed CCR with horizontal mirror using PZT cantilever actuator for large deflection at low induced voltage and supports for minimizing an initial tilting angle: (a) on-state and (b) off-state. 

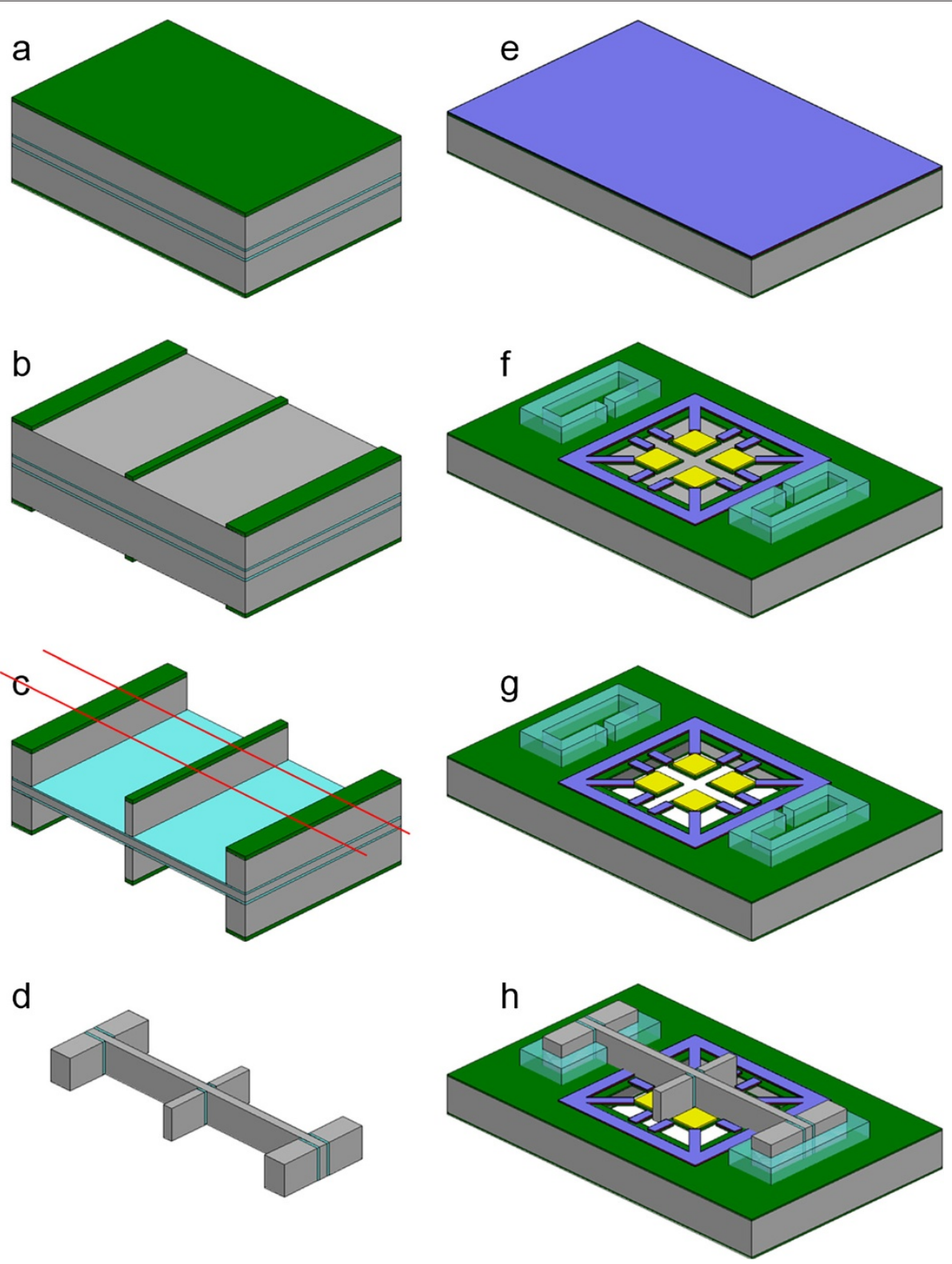

Figure 2 Fabrication sequences of proposed MEMS CCR with silicon cross shaped vertical mirrors and horizontal mirrors with PZT cantilevers: (a) deposition of SiNx on a double SOI wafer, (b) formation of mask for $\mathrm{KOH}$ wet etching, and (c) $\mathrm{KOH}$ wet etching, (d) dicing and rotation, (e) deposition of $\mathrm{SiNx} / \mathrm{Ti} / \mathrm{Pt} / \mathrm{PZT} / \mathrm{Pt}$ on a silicon wafer, (f) formation of PZT cantilevers, horizontal mirrors, torsional hinge springs, and SU-8 micro-holder, (g) KOH wet etching for release, and (h) dicing and assembly of the vertical mirrors onto the horizontal mirrors.

or $250 \times 250 \mu \mathrm{m}^{2}$ and torsional meander spring with 5 $\mu \mathrm{m}$ in width were formed by using low stress SiNx layer and Au was then deposited on the horizontal mirror by using lift-off technique. The SU-8 holders with thicknesses of $100 \mu \mathrm{m}$ were patterned to accurately align and hold the fabricated vertical mirror. Finally, the PZT cantilevers and the horizontal mirror were released by using $\mathrm{KOH}$ wet etching technique. The fabricated cantilevers have width and length of $70 \mu \mathrm{m}$ and $100 \mu \mathrm{m}$, respectively.
Finally, the proposed MEMS CCR was fabricated by aligning and inserting the vertical mirror manually into the micro-holder formed on the horizontal mirror as shown in Figure 2 (h).

Figure 3 shows scanning electron micrograph (SEM) pictures of the fabricated MEMS CCR. It is comprised of four CCRs where each device works independently. The fabricated vertical silicon mirror with $300 \mu \mathrm{m}$ (length) $\times$ $300 \mu \mathrm{m}$ (height) $\times 30 \mu \mathrm{m}$ (thickness) were well aligned 

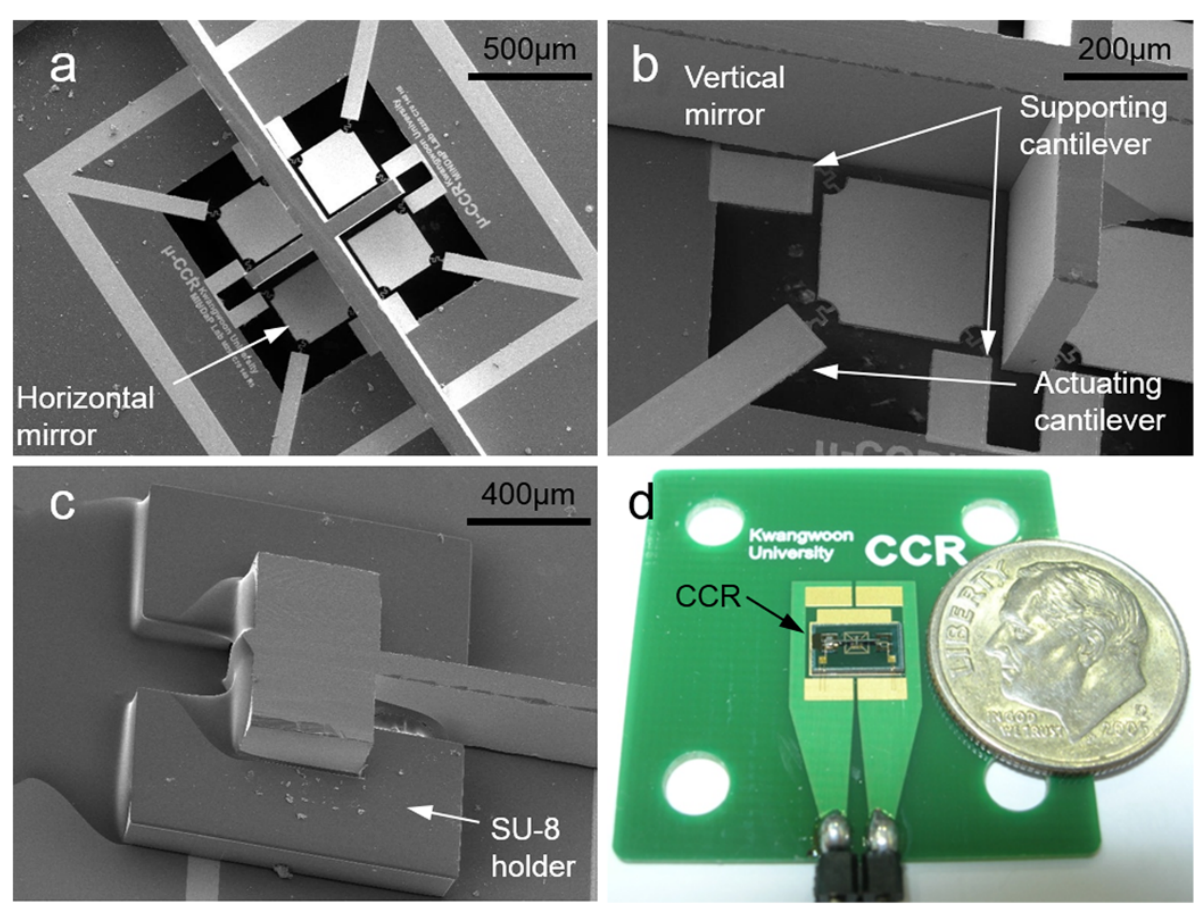

Figure 3 SEM pictures and photomicrograph of the fabricated MEMS CCR with two supporting and one actuating PZT cantilevers mounted on a PCB substrate: (a) top view of four MEMS CCRs, (b) side view of vertical mirror assembled on horizontal mirror with PZT cantilever, (c) SU-8 holder to align the vertical mirror, and (d) MEMS CCR on PCB test jig.

with the cross angle of $90^{\circ}$. The angular misalignment of two vertical mirrors measured by optical microscope was bounded within $0.32^{\circ}$ and the surface roughness measured by AFM was within $3.523 \mathrm{~nm}$ rms (rootmean-square). Figure 4 presents the surface topograph of fabricated horizontal mirrors. The surface roughness and radius of curvature of fabricated horizontal mirror measured by Nanofocus uSurf 3D non-contact profiler were approximately $5.75 \mathrm{~nm} \mathrm{rms}$ and $54.6 \mathrm{~mm}$, respectively. While the fabricated PZT cantilever exhibited large angular displacement of approximately $2.29^{\circ}$ with radius of curvature of $4.16 \mathrm{~mm}$ due to its residual stress, the angular misalignments $(\delta)$ were within $0.28^{\circ}$ and $0.13^{\circ}$ for the horizontal mirrors with area of $150 \times 150$ $\mu \mathrm{m}^{2}$ and $250 \times 250 \mu \mathrm{m}^{2}$, respectively, due to the use of two supporting cantilevers. The smaller angular misalignment can be achieved by optimizing the length of cantilevers or residual stress of PZT cantilevers. The horizontal mirror exhibited an angular misalignment below $0.05^{\circ}$ through the FEM simulation using Coventor-
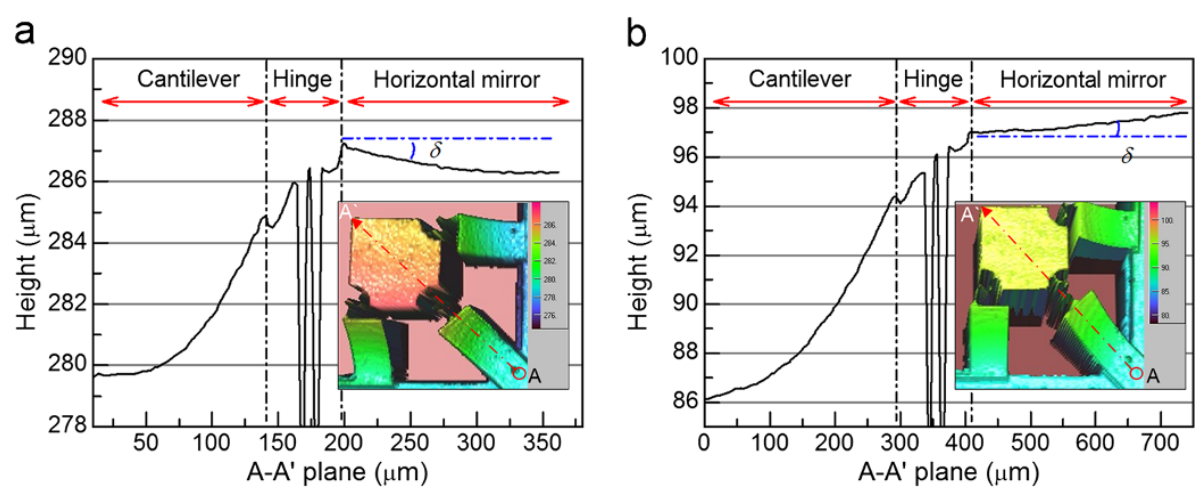

Figure 4 Surface topograph of fabricated horizontal mirrors using PZT cantilever actuator with area of $150 \times 150 \mu \mathrm{m}^{2}$ (a) and $250 \times$ $250 \mu \mathrm{m}^{2}$ (b) by using Nanofocus uSurf 3D non-contact profiler. 


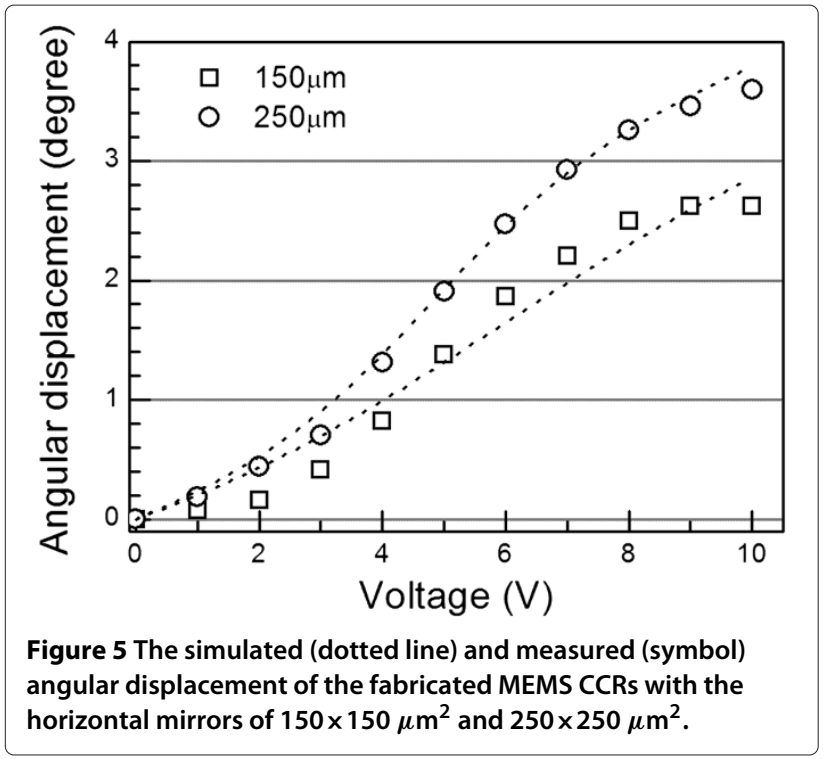

Ware at the overall residual stress of $200 \mathrm{MPa}$ in PZT cantilevers.

Figure 5 shows the angular displacement of the fabricated MEMS CCRs with two different horizontal mirrors. The fabricated CCRs with the horizontal mirrors of $150 \times 150 \mu \mathrm{m}^{2}$ and $250 \times 250 \mu \mathrm{m}^{2}$ exhibited the angular displacement of $1.37^{\circ}$ and $1.87^{\circ}$ at 5 volts, which are good enough for on-off keying, respectively. As shown in Figure 5, the measured angular displacement was also in good agreement with the simulated one.

The fabricated CCR was demonstrated to check the feasibility as a passive optical transmitter. Firstly, the reflective pattern was observed to confirm that the three mirrors were well aligned orthogonally to each other. Figure 6 (a) shows the reflective pattern of an unactuated CCR with diagonal illumination $(\lambda=632.8 \mathrm{~nm})$. As shown in Figure 5, the patterns exhibit similar "star" patterns due to six effective reflective regions [3]. Figure 6 (b) and (c) present the captured images by CMOS image sensor in

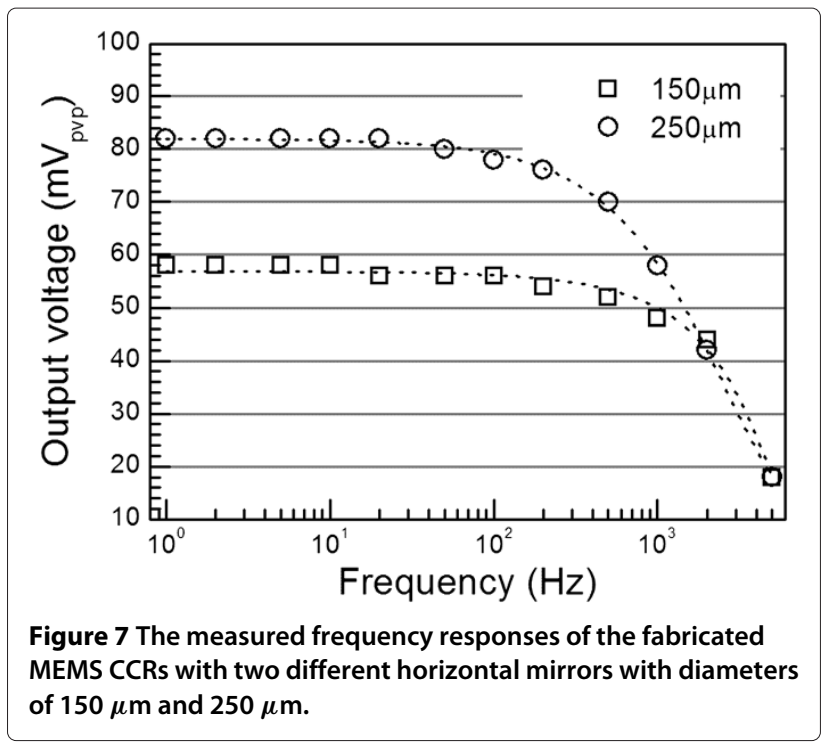

the off-state and on-state of the fabricated CCR. As shown in Figure 6 (b) and (c), there is a clear difference between the "on" and "off" states. In order to detect a He-Ne laser beam reflected from the CCR, a silicon photodiode was utilized as a receiver. The installed distance between the CCR and receiver was $50 \mathrm{~cm}$ because the fabricated CCR had large angular misalignment. As shown in Figure 7, the output voltage at the photodiode was significantly decreased as the driving frequency increases above $1 \mathrm{kHz}$. It might be attributed to the finite switching time, so that the mirror does not undergo full angular displacement and creates reduced peak-to-peak voltage. The 3-dB cutoff frequencies were approximately $2.5 \mathrm{kHz}$ and $1 \mathrm{kHz}$ for the CCR with the horizontal mirrors of $150 \times 150 \mu \mathrm{m}^{2}$ and $250 \times 250 \mu \mathrm{m}^{2}$, respectively. It also exhibited good switching characteristics with an off-to-on-state transition of $163 \mu$ s and on-to-off-state transition of $276 \mu$ s at a rectangular input voltage and switching frequency of $10 \mathrm{~V}$ and $1 \mathrm{kHz}$, respectively.
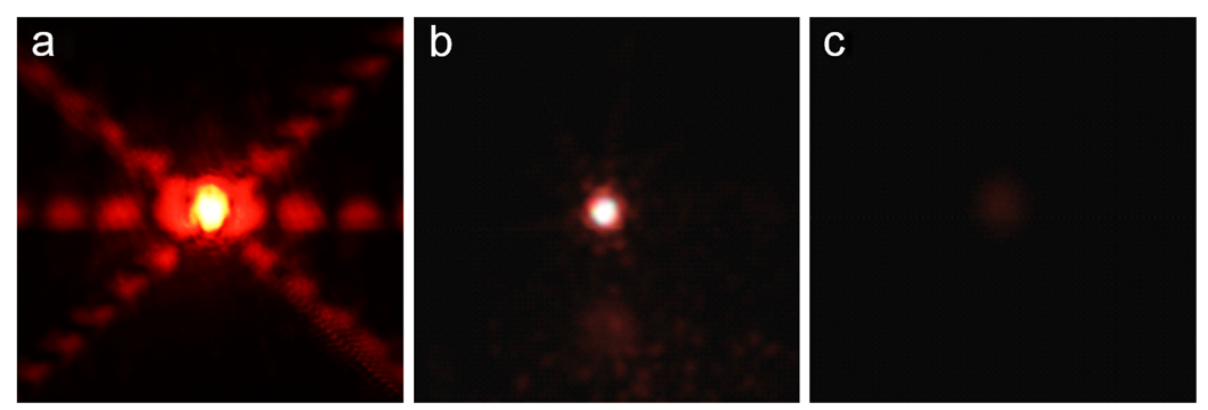

Figure 6 Photograph of the reflective pattern of the fabricated MEMS CCR from diagonally illuminated lay $(\lambda=632.8 \mathrm{~nm})(\mathrm{a})$, and captured images in off-state (b) and on-state (c) by using a CMOS image sensor. 


\section{Conclusions}

Silicon bulk micromachined CCRs have been presented for free space optical communications. They were comprised of two vertical silicon mirrors and one piezoelectrically actuating horizontal mirror. The fabricated vertical mirror exhibited an accurate angular alignment of three mutually orthogonal reflective surfaces by using anisotropic wet etching technique of (110) Si wafer. The fabricated horizontal mirror with PZT cantilever actuator exhibited large angular displacement and low switching voltage for on-off keying. The alignment of the horizontal mirror with the vertical mirror was significantly improved by applying the supporting PZT cantilevers and meander springs.

\section{Competing interests}

The authors declare that they have no competing interests.

\section{Authors' contributions}

JYP and JP carried out the design \& simulation and drafted the manuscript. JP carried out the fabrication of MEMS device and experimental measurements. Both authors read and approved the final manuscript.

\section{Acknowledgements}

The authors are grateful to acknowledge the support from the Basic Science Research Program (2010-0024618) through the National Research Foundation of Korea (NRF) funded by the Ministry of Education, Science and Technology, Korea.

Received: 28 September 2013 Accepted: 25 November 2013 Published: 18 December 2013

\section{References}

1. Kahn J, Katz RH, Pister K (2000) Emerging challenges: mobile networking for śmart dust: J Commun Netw 2:188-196

2. Chu PB, Lo NR, Berg EC, Pister KSJ (1997) Optical communication using micro corner cube reflectors In: Proceedings of IEEE Micro Electro Mechanical Systems Workshop, pp 350Ü355. IEEE, Piscataway, NJ

3. Zhou L, Kahn JM, Pister KSJ (2003) Corner-cube retrore ectors based on structure-assisted assembly for free-space optical communication. Jicroelectromech Syst 12:233-242

4. Hong YK, Syms RRA, Pister KSJ, Zhou LX (2005) Design, fabrication and test of self-assembled optical corner cube reflectors. J Microelectromech Syst 15:663-672

5. Zhu X, Hsu VS, Kahn JM (2002) Optical modeling of mems corner-cube retroreflectors with misalignment and nonflatness. IEEE J Select Top Quantum Electron 48:26-32

6. Park JC, Park JY, Y WJ, Kim DH, Park J (2011) Silicon bulkmicromachined piezoelectically actuated corner cube retro reflector In: 16 th Int Conf on Solid-State Sensors, Actuators and Microsystems, Transducers, pp 1578-1582. IEEE, Piscataway, NJ

doi:10.1186/2213-9621-1-7

Cite this article as: Park and Park: A bulk-micromachined corner cube retroreflector with piezoelectric micro-cantilevers. Micro and Nano Systems Letters 2013 1:7.

\section{Submit your manuscript to a SpringerOpen ${ }^{\mathcal{O}}$ journal and benefit from:}

- Convenient online submission

- Rigorous peer review

- Immediate publication on acceptance

- Open access: articles freely available online

- High visibility within the field

- Retaining the copyright to your article

Submit your next manuscript at $\boldsymbol{\wedge}$ springeropen.com 